\title{
Resultados do tratamento da ambliopia com levodopa combinada à oclusão
}

\author{
Resultsof amblyopia treatment with levodopa associated with occlusion therapy
}

\author{
Edson Procianoy ${ }^{1}$ \\ Letícia Procianoy ${ }^{2}$ \\ Fernando Procianoy ${ }^{3}$
}

Trabalho realizado no Setor de Estrabismo do Serviço de Oftalmologia do Hospital de Clínicas de Porto Alegre.

${ }^{1}$ Professor Chefe do Setor de Estrabismo do Serviço de Oftalmologia da Faculdade de Medicina da Universidade Federal do Rio Grande do Sul - UFRGS.

${ }^{2}$ Oftalmologista, Fellow do Setor de Estrabismo no Serviço de Oftalmologia do Hospital de Clínicas de Porto Alegre da Universidade Federal do Rio Grande do Sul - UFRGS.

${ }^{3}$ Médico Residente do Serviço de Oftalmologia do Hospital de Clínicas de Porto Alegre da Universidade Federal do Rio Grande do Sul - UFRGS.

Endereço para correspondência: Edson Procianoy Av. Carlos Gomes, 1200 / 805, Porto Alegre (RS)

CEP 91340-430 - E-mail: eprocianoy@terra.com.br

Recebido para publicação em 20.10.2003

Versão revisada recebida em 25.03.2004

Aprovação em 23.06.2004

\begin{tabular}{|c|}
\hline RESUMO \\
\hline $\begin{array}{l}\text { Objetivo: Verificar a melhora da acuidade visual com levodopa/benzerazida } \\
\text { combinada à oclusão parcial e seguida por oclusão total, em pacientes com } \\
\text { ambliopia consideradairreversível. Métodos: Realizou-se estudoexperimen- } \\
\text { tal aberto, envolvendo } 37 \text { pacientes entre } 7 \text { e } 40 \text { anos de idade, com ambliopia } \\
\text { por estrabismo ou anisometropia, durante } 9 \text { semanas. Todos os pacientes } \\
\text { foram tratados com levodopa }(0,70 \mathrm{mg} / \mathrm{kg} / \mathrm{dia} \text { ) e benzerazida } 25 \% \text { associada } \\
\text { à oclusão de } 4 \text { horas/dia do olho dominante por } 5 \text { semanas e, nas } 4 \text { semanas } \\
\text { seguintes foi realizada somente a oclusão total ( } 24 \text { h) do olho dominante. A } \\
\text { acuidade visual foi medida na tabela do ETDR (Early Treatment Diabetic } \\
\text { Retinopathy) com escala logMAR (logaritmo do mínimo ângulo de resolu- } \\
\text { ção) antes de iniciar o tratamento e após } 1,3,5 \text { e } 9 \text { semanas de tratamento. } \\
\text { As adesões ao tratamento de oclusão e a ingesta do me-dicamento foram } \\
\text { verificadas por meio de questionário e pela contagem das cápsulas. Os } \\
\text { efeitos adversos foram avaliados por exame clínico e questionário. Resul- } \\
\text { tados: Após } 9 \text { semanas de tratamento, a acuidade visual média melhorou em } \\
\text { logMAR de } 0,58 \pm 0,16 \text { para } 0,23 \pm 0,16 \text { (melhora de } 4 \text { linhas na tabelaETDR). } \\
\text { Conclusão: Levodopa, na dose de } 0,70 \text { mg/kg/dia, ébem tolerada associada } \\
\text { à oclusão produz melhora significativa na acuidade visual de pacientes com } \\
\text { ambliopia considerada irreversível. }\end{array}$ \\
\hline
\end{tabular}

Descritores: Acuidade visual; Ensaio clínico [tipo de publicação]; Ambliopia/etiologia; Ambliopia/quimioterapia; Levodopa/uso terapêutico; Estrabismos/complicações; Anisometropia/complicações

\section{INTRODUÇ̃̃̃O}

A ambliopia é a causa mais freqüente de perda visual na criança, afetando 2 a $5 \%$ da população ${ }^{(1)}$. Estrabismo e anisometropia são as causas mais frequientes de ambliopia. Embora se discuta a idade limite para se recuperar ambliopia, considera-se, sob o ponto de vista prático, irreversível a não tratada até os 8 anos de idade ${ }^{(2)}$.

A função das catecolaminas na plasticidade sensorial da visão já foi demonstrada em vários estudos ${ }^{(3-6)}$.

A levodopa é precursora da dopamina, um dos principais neurotransmissores do sistema extrapiramidal. Seu emprego tradicional é na doença de Parkinson, uma situação clínica classicamente atribuída à depleção de dopamina no estriado ${ }^{(4)}$. Pelo fato da dopamina não atravessar a barreira hemato-encefálica, o tratamento que intenta aumentar sua concentração no sistema nervoso central utiliza a levodopa, que transportada através da barreira, transforma-se em dopamina e subseqüentemente em norepinefrina. Como a levodopa também pode ser convertida em dopamina a nível periférico, o que ocasiona efeitos indesejáveis, administra-se carbidopa ou benzerazida simultaneamente para inibir esta transformação. 
Nos últimos anos vários trabalhos ${ }^{(1-20)}$ estudaram os efeitos da levodopa para melhorar a visão em pacientes com ambliopia. Alguns autores ${ }^{(16)}$ demonstraram melhora clínica e estatisticamente significativa da acuidade visual em pacientes tratados com a dose média de $0,51 \mathrm{mg} / \mathrm{kg} / \mathrm{dia}(0,38-0,68 \mathrm{mg} /$ $\mathrm{kg} / \mathrm{dia}$ ) de levodopa quando comparada com placebo. Esta dose média era aproximadamente $1 / 3$ da recomendada como efetiva na literatura. Este estudo foi o único a apresentar um delineamento em paralelo, testando o efeito de 3 doses. Outros autores ${ }^{(11)}$, mostraram que há vantagem de se usar levodopa por mais de uma semana, mas não por mais de 5 semanas, e que levodopa associada à oclusão parcial ( $3 \mathrm{~h} / \mathrm{dia})$ é mais efetiva que o uso isolado de levodopa ${ }^{(15)}$. Até o momento, os trabalhos realizados que demonstraram os benefícios da levodopa associada à oclusão, não mostram os resultados da oclusão de 24 horas após o uso do medicamento.

O objetivo deste estudo é verificar a melhora da acuidade visual, em pacientes com ambliopia considerada irreversível, com o tratamento de levodopa/benzerazida combinada com a oclusão parcial do olho dominante e seguida de um período de oclusão total.

\section{MÉTODOS}

Foram estudados pacientes entre 7 e 40 anos, com ambliopia por estrabismo ou anisometropia no período de junho de 1999 a junho de 2003. As características basais dos pacientes são apresentadas na tabela 1. Os pacientes estudados já haviam feito oclusão total e eram considerados amblíopes que não respondiam mais com a terapia de oclusão ou penalização. A acuidade visual do olho amblíope variou em logaritmo do mínimo ângulo de resolução $(\log M A R)$ de 0,3 a $1(0,5$ a 0,1 na escala Snellen). Os pacientes foram recrutados do ambulatório de estrabismo do Hospital de Clínicas de Porto Alegre e da Clínica do autor. O estudo foi aprovado pelo Comitê de Ética da instituição.

\section{Delineamento e procedimento}

Realizou-se um estudo experimental aberto por 9 semanas. Nas primeiras 5 semanas os pacientes foram instruídos a fazer oclusão do olho dominante de $4 \mathrm{~h} / \mathrm{dia}$ e a tomar $0,70 \mathrm{mg} / \mathrm{kg} / \mathrm{dia}$ de levodopa com $1 / 4$ de benzerazida divididas em 3 doses diárias, que foram sempre administradas após as refeições. Levodopa/benzerazida na proporção de $4: 1$, foram preparadas em

\begin{tabular}{|lcc|}
\hline \multicolumn{2}{|c|}{ Tabela 1. Características basais dos pacientes } \\
Variáveis & $\begin{array}{c}\text { Ambliopia- } \\
\text { Estrabismo } \\
(\mathbf{n}=\mathbf{2 9})\end{array}$ & $\begin{array}{c}\text { Ambliopia- } \\
\text { Anisometropia } \\
(\mathbf{n}=\mathbf{8})\end{array}$ \\
Sexo masculino & $16(59,3 \%)$ & $2(28,6 \%)$ \\
Peso (kg) & $36,17 \pm 16,87$ & $47,75 \pm 14,46$ \\
Idade (anos) & $10,79 \pm 4,88$ & $17,50 \pm 12,17$ \\
\hline
\end{tabular}

cápsulas por um laboratório referido por nossa Universidade. Nas quatro semanas seguintes foram orientados a fazer oclusão total $(24 \mathrm{~h} / \mathrm{dia})$ do olho dominante. Como estes pacientes também faziam parte de uma pesquisa na qual verificou-se o tempo ideal de uso da levodopa, a acuidade visual foi avaliada após 1, 3 e 5 semanas nas primeiras cinco semanas. A acuidade visual final foi avaliada ao término das quatro semanas de oclusão total. A adesão ao tratamento foi verificada pela contagem de cápsulas que restavam nos frascos e por questionário respondido pelo paciente ou pelos pais quando menor de 18 anos.

Os pacientes foram submetidos a exame clínico e oftalmológico antes e após o tratamento. A acuidade visual foi verificada em $\log$ MAR, com as cartas do ETDR (Early Treatment Diabetic Retinopathy Study/ Lighthouse for the Blind, New York), na mesma sala e com a mesma luminosidade (sala escura com iluminação somente dos optotipos). Para evitar a memorização das letras, o olho amblíope foi sempre testado primeiro. A acuidade visual foi medida com correção óptica e com estenopeico sobre a correção, registrando-se a melhor acuidade. Os pacientes foram estimulados, a fim de obter a melhor acuidade visual, apontando-se cada optotipo. Considerou-se que a acuidade visual era a da linha em que o paciente conseguia ler mais da metade dos optotipos. O exame foi realizado sempre pelo mesmo médico.

\section{Efeitos adversos}

A ocorrência de efeitos adversos como náuseas, vômitos, anorexia, epigastralgia, hipotensão postural e alterações do humor foram sistematicamente verificadas pelo exame clínico.

\section{Análise}

Todas as estatísticas foram feitas com o logaritmo do mínimo ângulo de resolução (logMAR) empregando-se testes paramétricos (ANOVA). Inicialmente participaram 60 pacientes, dos quais foram excluídos 20 por terem sido considerados insensíveis ao tratamento (melhora inferior a uma linha após uma semana de tratamento).

O critério de exclusão destes pacientes baseou-se na experiência do trabalho de Procianoy e cols. ${ }^{(16)}$ com levodopa. Neste estudo, ao final de 7 dias, a acuidade visual melhorou pelo menos uma linha em $40-68,2 \%$ dos pacientes que receberam levodopa associada à oclusão de $3 \mathrm{~h}$ por dia. Foram também excluídos da análise os pacientes que não fizeram a oclusão recomendada ou deixaram de tomar o medicamento por mais de um dia, restando um total de 37 pacientes.

\section{RESULTADOS}

Todos os pacientes, exceto dois casos, realizaram a oclusão recomendada. A adesão à medicação só não aconteceu com um paciente. Não foram observados efeitos adversos ao longo de todo o estudo. 


\begin{tabular}{|c|c|c|c|}
\hline $\begin{array}{l}\text { Acuidade } \\
\text { Visual } \\
\text { (LogMAR) }\end{array}$ & $\begin{array}{c}\text { Ambliopia - } \\
\text { Estrabismo } \\
+ \\
\text { Ambliopia - } \\
\text { Anisometropia } \\
(n=37)\end{array}$ & $\begin{array}{c}\text { Ambliopia - } \\
\text { Estrabismo } \\
(n=29)\end{array}$ & $\begin{array}{c}\text { Ambliopia - } \\
\text { Anisometropia } \\
(n=8)\end{array}$ \\
\hline Basal & $0,58 \pm 0,16$ & $0,57 \pm 0,16$ & $0,60 \pm 0,20$ \\
\hline 9 semanas & $0,23 \pm 0,16$ & $0,25 \pm 0,16$ & $0,18 \pm 0,14$ \\
\hline
\end{tabular}

A tabela 2 mostra a acuidade visual basal e após 9 semanas de tratamento, expressa em logMAR, nos 37 pacientes e, separadamente, conforme a causa da ambliopia. Não houve diferença significativa na melhora da acuidade visual entre os grupos com ambliopia anisometrópica e por estrabismo.

A acuidade visual média, em logMAR, ao final de 5 semanas melhorou de 0,58 (0,25 Snellen) para 0,37 (0,40 Snellen) $(\mathrm{p}<0,001)$ e após as 4 semanas de oclusão total melhorou para 0,23 (0,66 Snellen) $(\mathrm{p}<0,001)$.

Ao final das 9 semanas a acuidade visual melhorou em média 4 linhas, ficando 0,3 logMAR (0,5 Snellen), ou melhor, em $78,3 \%$ dos 37 pacientes. Em três pacientes $(8,1 \%)$, a acuidade visual ficou 0,0 logMAR (1 Snellen).

\section{DISCUSSÃO}

Os resultados do nosso estudo mostraram uma melhora estatística e clinicamente significativa da acuidade visual em pacientes que eram considerados amblíopes irreversíveis.

Após 5 semanas de $0,70 \mathrm{mg} / \mathrm{kg} / \mathrm{dia}$ de levodopa associada à oclusão de $4 \mathrm{~h} /$ dia houve melhora média na acuidade visual de $37,5 \%$. Leguire e cols. ${ }^{(11)}$, empregando $1,65 \mathrm{mg} / \mathrm{kg} / \mathrm{dia}$ de levodopa associada à oclusão de $3 \mathrm{~h} /$ dia, obtiveram uma melhora de $37 \%$ ao final da quinta semana.

Nossos resultados com a oclusão total após uso de levodopa por 5 semanas corroboram com o estudo de outros autores $^{(11)}$ que mostrou a manutenção dos efeitos da levodopa após parada de sua ingestão. Nosso estudo é o único a quantificar a melhora da acuidade visual com a oclusão total (24 h) de 4 semanas após emprego de levodopa associada com oclusão parcial. É também, o estudo que inclui o maior número de pacientes que fizeram oclusão parcial combinada com levodopa por 5 semanas.

\section{CONCLUSÃO}

Considerando-se que a acuidade visual de 0,3 logMAR (0,5 escala de Snellen) em cada olho é suficiente para obter-se carteira de direção nos Estados Unidos, e que a acuidade de 1 logMAR (0,1 escala Snellen) é considerado cegueira legal, nossos resultados mostram que a levodopa associada com a oclusão pode ser um tratamento valioso para pacientes consi- derados amblíopes irreversíveis. A melhora da acuidade visual de nossos pacientes, que eram considerados amblíopes irreversíveis, fortalece a idéia de que a levodopa restabelece a plasticidade sensorial da visão. Verificar a manutenção destes resultados será importante para a consagração do uso da levodopa no tratamento da ambliopia.

\section{ABSTRACT}

Purpose: To evaluate visual acuity improvement with levodo$\mathrm{pa} /$ benzerazide associated with partial occlusion and followed by total occlusion therapy in patients with amblyopia considered irreversible. Methods: A 9-week experimental open study was performed involving 37 patients, between 7 and 40 years old, with strabismic and/or anisometropic amblyopia. All patients were treated with levodopa $(0.70 \mathrm{mg} / \mathrm{kg} /$ day $)$ and $25 \%$ benzerazide associated with 4-hour/day occlusion of the dominant eye for 5 weeks. In the last 4 weeks, only the total occlusion $(24 \mathrm{~h})$ of the dominant eye was performed. Visual acuity was measured by the Early Treatment Diabetic Retinopathy (ETDR) table with the logarithmic minimum angle of resolution ( $\log$ MAR) scale before the beginning of the treatment and after 1, 3, 5 and 9 weeks of treatment. Adhesions to occlusion therapy and to drug intake were checked through a written questionnaire and capsule counting. Adverse effects were evaluated by clinical examination and questionnaire. Results: After 9 weeks of treatment visual acuity improved from $0.58 \pm 0.16$ to $0.23 \pm 0.16 \log$ MAR (4 lines of improvement in the ETDR table). Conclusion: Levodopa dose of $0.70 \mathrm{mg} /$ $\mathrm{kg} /$ day is well tolerated and, when associated with occlusion therapy, significantly improves visual acuity in patients with amblyopia considered irreversible.

Keywords: Visual acuity; Clinical Trial [publication type]; Amblyiopia/etiology; Amblyopia/drug therapy; Levodopa/ therapeutic use; Strabismus/complications; Anisometropia/ complications

\section{REFERÊNCIAS}

1. Simons K, Reineke RD. Amblyopia screening and stereopsis. In: Symposium on strabismus. St. Louis: CV Mosby; 1978. p.15-50.

2. Greenwald MO, Parks MM. Amblyopia. In: Duane TD, editor. Clinical ophthalmology. Philadelphia: Lippincott; 1992. vol.1

3. Kasamatsu T. Enhancement of neuronal plasticity by activating the norepinephrine system in the brain: a remedy for amblyopia. Hum Neurobiol. 1982;1 (1):49-54.

4. Fuchs FD, Wannmacher L. Antiparkinsonianos. In: Fuchs FD, Wannmacher L Farmacologia clínica. Rio de Janeiro: Guanabara Koogan; 1992. p.309-21.

5. Kasamatsu T, Imamura K. Ocular dominance plasticity in kitten visual cortex: integration of noradrenergic and cholinergic regulation. In: Richardson RT, editor. Activation to acquisition: functional aspects of the basal forebrain cholinergic system. Boston: Birkhauser; 1991. p.289-324.

6. Kasamatsu T, Heggelund P. Single cell responses in cat visual cortex to visual stimulation during iontophoresis of noradrenaline. Exp Brain Res. 1982:45(3):317-27. 
7. Gottlob I, Stangler-Zuschrott E. Effect of levodopa on contrast sensitivity and scotomas in human amblyopia. Invest Ophthalmol Vis Sci. 1990;31(4):776-80.

8. Bartholini G, Pletscher A. Cerebral accumulation and metabolism of C14dopa after selective inhibition of peripheral decarboxylase. J Pharmacol Exp Ther. 1968;161(1):14-20.

9. Leguire LE, Rogers GL, Bremer DL, Walson PD, McGregor ML. Levodopa/ carbidopa for childhood amblyopia. Invest Ophthalmol Vis Sci. 1993;34(11): 3090-5.

10. Gottlob I, Wizov SS, Reinecke RD. Visual acuities and scotomas after 3 weeks' levodopa administration in adult amblyopia. Graefes Arch Clin Exp Ophthalmol. 1995;233(7):407-13.

11. Leguire LE, Walson PD, Rogers GL, Bremer DL, McGregor ML. Levodopa/ carbidopa treatment for amblyopia in older children. J Pediatr Ophthalmol Strabismus. 1995;32(3):143-51.

12. Gottlob I, Charlier J, Reinecke RD. Visual acuities and scotomas after one week levodopa administration in human amblyopia. Invest Ophthalmol Vis Sci. 1992;33(9):2722-8.

13. Leguire LE, Rogers GL, Bremer DL, Walson P, Hadjiconstantinou-Neff M. Levodopa and childhood amblyopia. J Pediatr Ophthalmol Strabismus. 1992;29(5):290-8.
14. Leguire LE, Walson PD, Rogers GL, Bremer DL, McGregor ML. Longitudinal study of levodopa/carbidopa for childhood amblyopia. J Pediatr Ophthalmol Strabismus. 1993;30(6):354-60.

15. Leguire LE, Walson PD, Rogers GL, Bremer DI, McGregor ML. Occlusion and levodopa-carbidopa treatment for childhood amblyopia. J AAPOS. 1998;2(5):257-64

16. Procianoy E, Fuchs FD, Procianoy L, Procianoy F. The effect of increasing doses of levodopa on children with strabismic amblyopia. J AAPOS. 1999;3 (6):337-40.

17. Mohan K, Khankar V, Sharma A. Visual acuities after levodopa administration in amblyopia. J Pediatr Ophthalmol Strabismus. 2001;38(2):62-7.

18. Pandey PK, Chaudhuri Z, Kumar M, Satyabala K, Sharma P. Effect of levodopa and carbidopa in human amblyopia. J Pediatr Ophthalmol Strabismus. 2002;39 (2):81-9.

19. Bhartiya P, Sharma P, Biswas NR, Tandon R, Khokhar SK. LevodopaCarbidopa with occlusion in older children with amblyopia. J AAPOS. 2002; 6(6):368-72.

20. Leguire LE, Komaromy KL, Nairus TM, Rogers GL. Long-term follow-up of L-dopa treatment in children with amblyopia. J Pediatr Ophthalmol Strabismus. 2002;39(6):326-30.

\section{FÓRUM 2005 DO INSTITUTO PENIDO BURNIER}

\section{2 a 5 de Junhe de 2005 The Royal Palm Plaze. Compinas - SP}

\section{Tema: Cirurgia da Catarata}

INFORMAÇÕES: Tel.: (19) 3232-5866 - Ramal 2147

Fax: (19) 3233-4492

E-mail: penido@penidoburnier.com.br 